\title{
Impacto do Crescimento, da Concentração da Renda e das Transferências Governamentais sobre a Pobreza nos Municípios Brasileiros
}

\section{The Impact of Income Growth, Inequality and Government Transferences over Brazilian Municipalities' Poverty}

Cristiano Aguiar de Oliveira* Francisco Soares de Lima**

Resumo: Este artigo estima as elasticidades-renda (advinda do trabalho e das transferências governamentais) e a elasticidade-concentração da renda em relação à pobreza nos municípios brasileiros nos anos de 1991 e de 2000. As elasticidades são calculadas a partir dos coeficientes obtidos por regressão quantílica. Observou-se que as maiores elasticidades são da concentração, depois a elasticidade da renda do trabalho e, finalmente, a elasticidade-renda de transferências governamentais, que apresenta valores muito baixos. As elasticidadesrenda e concentração diminuíram entre os anos 1991 e 2000, principalmente nos quantis com maior incidência de pobres. Por outro lado, a elasticidade-renda das transferências governamentais aumentou nos municípios com mais pobres, o que implica que este tipo de política tornou-se relativamente mais eficiente na redução da pobreza. $\mathrm{O}$ artigo conclui que políticas de combate à pobreza devem ser implementadas considerando as diferentes características apresentadas pelas distribuições de renda nos municípios brasileiros.

Palavras-chave: Pobreza. Crescimento econômico. Regressão quantílica.

Abstract: This paper estimates the income (from work and government transferences) elasticity and the income inequality elasticity with respect to poverty in Brazilian municipalities in 1991 and 2000. The elasticities are calculated using quantile regression. It was observed that the income inequality elasticities are bigger than income elasticity, which is bigger than government income transference elasticity, which shows low values. The income and income inequality elasticity have been diminishing during the period. Mainly in the quantiles with a higher incidence of poor people. On the other hand, the government income transference grew in municipalities with more poor people in the same period, which implies that this politic tool had become relatively more efficient in poverty reduction.

\footnotetext{
* Doutorando em economia aplicada PPGE/UFRGS. E-mail: cristiano.oliveira@ufrgs.br

** Doutor em economia. Professor da UERN. E-mail: fsoareslima@uol.com.br
} 
The paper concludes that poverty reduction policies should consider the existent differences presented by Brazilian municipalities' income distribution.

Keywords: Poverty. Economic growth. Quantile regression.

JEL Classification: C21; I32; I38.

\section{Introdução}

Dos possíveis indicadores de bem-estar social, a ausência de pobreza certamente é um dos mais aceitos. Em todas as sociedades, buscam-se meios de reduzir ou acabar com a existência de pessoas em condições sociais abaixo de um mínimo considerado aceitável. Estudos recentes mostram que a pobreza está associada a distúrbios de violência e saúde pública, entre outros fatores de perda de bem-estar. Portanto, implementar políticas para reduzir a pobreza pode resolver outros problemas indiretamente.

Recentemente, o País reforçou a sua intenção de utilizar políticas de transferências de renda como uma forma de reduzir a pobreza e melhorar a distribuição da renda. Entretanto, existem poucos estudos a respeito dos impactos deste tipo de política. Até então, o que se tinha era a ideia de que a promoção do crescimento econômico seria uma estratégia recomendável de combate à pobreza. Implicitamente, supõe-se a existência de uma relação negativa entre as duas variáveis. No entanto, as evidências da relação negativa entre crescimento e pobreza também mostram que há diferentes níveis de respostas da pobreza em relação ao crescimento. Estudos recentes têm procurado explicar quais fatores estão influenciando a elasticidade renda-pobreza nos países em desenvolvimento.

Ravallion e Datt (1999), analisando regiões da Índia, encontraram que no setor não agrícola há elasticidades renda-pobreza significativamente diferentes entre regiões e que as elasticidades são maiores nas regiões onde a produtividade agrícola, o padrão de vida no meio rural e a escolaridade são maiores.

Chen e Wang (2001) investigaram a relação entre a pobreza, a renda e a desigualdade na China nos anos 90. A variação na pobreza foi decomposta em dois termos correspondendo à variação na renda média e à mudança na concentração de renda. Concluíram que o crescimento da renda reduziu significativamente a pobreza e a concentração contribuiu para aumentá-la. Outra conclusão do estudo foi que o crescimento econômico beneficiou relativamente os ricos. Especificamente, verificou-se que apenas a renda média dos $20 \%$ mais ricos cresceu mais do que a renda média total. Este último resultado é uma evidência de que a concentração da renda pode reduzir o efeito do crescimento sobre a pobreza.

No Brasil, Barros e Mendonça (1997) estimaram o efeito do crescimento econômico e da redução da desigualdade sobre a pobreza. Eles 
mostraram que o impacto da redução da concentração de renda sobre a pobreza é significativamente maior do que o impacto do crescimento econômico.

Marinho e Soares (2003) estimam as elasticidades renda-pobreza e concentração-pobreza para os estados brasileiros usando dados em painel. Foi estimado um modelo que permitisse a variação das elasticidades no tempo e também entre as unidades da federação componentes da amostra. Verificou-se que quanto maior a renda média, maior é o impacto negativo da distribuição da renda sobre a pobreza. De modo geral, o trabalho mostra que políticas de distribuição de renda mostram-se mais eficientes no curto prazo para reduzir a pobreza. Hoffman (2005) chega às mesmas conclusões destes autores, entretanto, calcula as elasticidades a partir de uma distribuição log-normal da renda dos estados brasileiros.

Se por um lado existem resultados coincidentes com relação aos impactos da desigualdade de renda e do crescimento da renda na pobreza, sendo o primeiro positivo e o segundo negativo, por outro lado, existem discrepâncias quanto à magnitude destes impactos. Em princípio espera-se que cada unidade região estudada possua uma distribuição de rendimentos própria e que estes impactos sejam distintos. Portanto, existe uma heterogeneidade não observada em trabalhos que utilizam dados nacionais ou estaduais, uma vez que estas unidades apresentam uma grande heterogeneidade interna.

Este artigo tenta contribuir para a discussão deste tema apresentando uma formulação alternativa que reduz o problema de heterogeneidade através da combinação de dados municipais e regressões quantílicas. Desta forma, para avaliar os impactos do crescimento econômico e da desigualdade de renda na redução da pobreza, este artigo estima as elasticidades renda-pobreza e concentração-pobreza que medem, respectivamente, o efeito de uma variação percentual da renda média (oriunda do trabalho ou de transferências governamentais) e da concentração de renda sobre a incidência de pobreza nos municípios brasileiros nos anos de 1991 e 2000. Estas elasticidades são obtidas através de regressões quantílicas.

Ao utilizar dados municipais, têm-se dois objetivos. O primeiro está relacionado à possibilidade de avaliar em uma grande amostra tais impactos, que até então só eram medidos com dados estaduais. A utilização de dados municipais permite uma análise diferenciada para o contexto de cada município, uma vez que permite observar como as políticas públicas de combate à pobreza podem gerar impactos diferentes em regiões e municípios com distribuições de renda distintas. Com a finalidade de ilustrar tais diferenças, o artigo faz uma breve avaliação das variações na pobreza dos principais municípios brasileiros na década de noventa. Por sua vez, a utilização de regressões quantílicas permite, por exemplo, captar as pos- 
Síveis diferenças nas elasticidades entre municípios com maior ou menor incidência de pobreza.

Este artigo está organizado da seguinte forma: na seção 2, é feita uma breve discussão a respeito das medidas de pobreza mais utilizadas em trabalhos empíricos e sobre a metodologia de regressões quantílicas. Na seção 3, serão descritas as variáveis utilizadas nas estimações e suas respectivas fontes, além disso, serão apresentados os principais resultados das estimações e decomposições da variação da pobreza. A seção apresenta ainda um maior detalhamento dos resultados e suas implicações para os principais municípios do Brasil. Na seção 4, são apresentadas as considerações finais.

\section{Metodologia}

\subsection{Medidas de Pobreza}

As medidas de pobreza normalmente encontradas na literatura podem ser classificadas em medidas monetárias e não monetárias. As medidas não monetárias definem pobreza como a insatisfação de necessidades básicas. Esta abordagem considera essencial o acesso a alguns bens sem os quais os indivíduos não gozariam de uma vida minimamente digna. Água potável, rede de esgoto, coleta de lixo, acesso ao transporte coletivo e educação são bens considerados imprescindíveis para que os indivíduos possam levar vidas saudáveis. Mesmo com alguma dificuldade, as Necessidades Básicas Insatisfeitas (NBI) são mensuráveis. Porém, há alguma arbitrariedade no cálculo do indicador, uma vez que é necessário estipular quais são as necessidades básicas e quais são as quantidades mínimas para as mesmas.

Existem outras medidas propostas que consideram a situação autoavaliada, ou seja, como o indivíduo percebe sua própria situação social. Esta vertente, conhecida como abordagem das capacidades, define a pobreza relativamente à capacidade dos indivíduos de exercerem suas liberdades bem como de fazerem respeitar seus direitos e busca analisar as diferentes formas de distribuição e acesso aos recursos privados e coletivos. É importante enfatizar não apenas os direitos sociais, mas também os direitos civis e políticos (SEN, 1988). Um indicador de pobreza com estas características pode ser chamado de relativo e subjetivo, ao considerar o sentimento de pobreza percebido pelos indivíduos. Entretanto, quando o objetivo é comparar a pobreza entre países ou regiões, esta percepção de pobreza pode produzir problemas insolúveis. A percepção da pobreza depende do que o indivíduo considera ser pobre. Este conceito pode ser diferente entre indivíduos de diferentes níveis sociais, regiões, escolaridade etc. 
Por sua vez, as medidas monetárias estão presentes na maioria dos trabalhos sobre fome e pobreza no Brasil e no resto do mundo. Em geral, trata-se de estudos que buscam aferir a pobreza e a fome através de indicadores de renda ou daqueles que buscam associar esta variável a outras fontes de dados existentes. A metodologia mais comum é considerar pobres os membros de famílias com renda per capita inferior a uma determinada linha de pobreza. Apesar desta metodologia sempre envolver algum grau de arbitrariedade ela é muito utilizada por permitir o cálculo do número de indivíduos pobres a partir de uma distribuição de renda e, além disso, por permitir que sejam feitas comparações entre países, estados, municípios etc.

A distribuição da renda entre as famílias ou indivíduos pode ser representada por uma distribuição de frequência ou uma função densidade de probabilidade, caso esta exista. Como toda distribuição de probabilidade teórica, a distribuição da renda depende dos seus parâmetros, tais como a renda média, a variância e a medida de assimetria. Desta forma, denota-se a função de densidade da renda por $f(y)$, onde $y$ é o nível de renda do indivíduo. Esta função é o primeiro passo para a definição de uma medida de pobreza.

Com a função de distribuição da renda pode-se construir uma medida de pobreza, bastando estabelecer uma linha de pobreza. Uma medida bastante comum em trabalhos empíricos é a medida pertencente à classe proposta por Foster, Greer e Thorbecke (1984) que é definida a partir da seguinte expressão:

$$
P_{\alpha}=\int_{0}^{L P}\left(\frac{L P-y}{L P}\right)^{\alpha} f(y) d y
$$

onde LP é a linha de pobreza. Esta linha de pobreza é sempre um valor de referência, para determiná-la existem diversas abordagens. Alguns autores simplesmente utilizam o valor correspondente a US $\$ 1 /$ dia ou US $\$ 2 /$ dia. Entretanto, esta pode ser definida como um valor de referência determinado pela renda mínima suficiente para adquirir os bens necessários à sobrevivência ou para a aquisição de uma cesta de bens de primeira necessidade.

A partir da expressão (1), pode-se derivar diversas medidas de pobreza, sendo as mais utilizadas $\mathrm{P}_{0}, \mathrm{P}_{1}$ e $\mathrm{P}_{2}$, para os valores de alfa $=0,1$ e 2 , respectivamente. Com o alfa igual a zero, tem-se a medida de incidência da pobreza que é simplesmente a razão entre o número de indivíduos vivendo em situação de pobreza e a população total ou simplesmente o percentual de pobres numa determinada economia. Esta é a medida utilizada neste artigo devido a sua disponibilidade e sua facilidade de interpretação.

Ao examinar detalhadamente a medida $\mathrm{P}_{0}$, é possível observar que a medida de pobreza depende exclusivamente dos parâmetros de $f(y)$. Uma variação em $\mathrm{P}_{0}$ pode ser resultado de uma variação na média e/ou 
nos demais momentos da distribuição. Considerando que a concentração de renda é função da variância e da assimetria de $f(y),{ }^{1}$ pode-se parametrizar $f(y)$ em termos da renda média e da medida de concentração e, portanto, uma variação percentual na pobreza pode ser decomposta pela variação percentual na renda média (W) e na medida de concentração (G) da seguinte forma: ${ }^{2}$

$$
\frac{d P_{0}}{P_{0}}=\varepsilon_{W} \frac{d W}{W}+\varepsilon_{G} \frac{d G}{G}
$$

onde $\varepsilon_{\mathrm{w}}$ representa a elasticidade-renda com relação à pobreza e $\varepsilon_{\mathrm{G}}$ representa a elasticidade-concentração com relação à pobreza. Estas podem ser obtidas de várias maneiras. O cálculo das elasticidades renda e elasticidades concentração da renda com relação à pobreza podem ser vistos em outros trabalhos, mas a maneira mais comum é a estimação destas elasticidades através de modelos econométricos.

O objetivo principal do artigo é a estimação destas elasticidades, entretanto, diferentemente dos trabalhos anteriores, estas serão estimadas com a utilização de regressões quantílicas. Antes disso, será feita uma breve explanação sobre a estimação com regressões quantílicas.

\subsection{Regressão Quantlica}

A utilização de regressões quantílicas apresenta algumas vantagens sobre o modelo tradicional de Mínimos Quadrados Ordinários (MQO). Em primeiro lugar, do ponto de vista teórico, é difícil imaginar que municípios tão heterogêneos possuam elasticidades idênticas. Certamente a sua posição na distribuição altera os parâmetros estimados e, por consequência, as suas elasticidades da renda e da concentração da renda sobre a pobreza. Em segundo lugar, as estimações na média de $\mathrm{MQO}$ tendem a dar um peso excessivo a observações discrepantes na explicação da variável dependente. Por sua vez, a regressão quantílica lida melhor com observações discrepantes, fornecendo estimativas mais robustas. ${ }^{3}$ Em terceiro lugar, as estimações de $\mathrm{MQO}$ assumem que o termo de erro possui a mesma distribuição, independente das variáveis explicativas, o que nem sempre ocorre. Umm modelo de $\mathrm{MQO}$ permite apenas a mudança de local na distribuição, enquanto a regressão quantilica permite alterações na curtose e na dispersão, pois não especifica nenhuma distribuição para o erro a priori. A regressão quantilica permite estimar toda a distribuição condicional da variável dependente uma vez que permite estimar os parâmetros em um

Conforme pode ser visto em Hoffman (1998).

Ver Kakwani (1993) para uma formalização desta decomposição.

Ver Koencker e Bassett (1978). 
intervalo contínuo entre zero e um. Além disso, esta metodologia permite calcular o impacto das variáveis explicativas sobre a variável dependente ao longo da distribuição condicional desta última, enquanto a estimação por MQO nos fornece apenas o efeito dos regressores na média condicional da variável dependente.

Um modelo de regressão linear quantílica com dados cross-section pode ser escrito como:

$$
y_{i}=x_{i} \beta+\xi_{i}, \text { para } i=1, \ldots, n \text { e } \tau[0,1]
$$

onde $y$ é a variável dependente, $x$ é uma matriz de variáveis covariadas, $\beta$ é o vetor de parâmetros a serem estimados, $\xi$ é o erro com uma distribuição que não necessariamente é conhecida e $\tau$ é o coeficiente do $\tau$-ésimo quantil condicional de $y$ dado $x$. A estimação pela regressão quantílica no intervalo $0<\tau<1$ será a minimização da seguinte função:

$$
\left.\min _{\beta \in \Re^{K}}\left|\sum_{i \in\left\{i: y_{i} \geq x_{i} \beta\right\}} \tau\right| y_{i}-x_{i} \beta\left|+\sum_{i \in\left\{i: y_{i}<x_{i} \beta\right\}}(1-\tau)\right| y_{i}-x_{i} \beta \mid\right\rfloor
$$

Diferentemente do modelo de MQO que minimiza o quadrado dos resíduos (a variância dos mesmos), a função objetivo ${ }^{4}$ minimiza os desvios absolutos de forma ponderada e, assim, pode ser interpretada como uma função de penalidade linear assimétrica. Vale salientar que os parâmetros estimados neste problema de minimização são consistentes e assintoticamente normais sob hipóteses adicionais de regularidade (BUCHINSKY, 1998). A interpretação dos parâmetros estimados em cada quantil pode ser feita da seguinte maneira: representa o impacto marginal no $\tau$-ésimo quantil condicional devido a uma mudança no i-ésimo elemento de $x$.

\subsection{Especificação do Modelo e Fonte dos Dados}

Para estimar o modelo proposto na equação 2, utilizaram-se as variáveis referentes à incidência de pobreza $\left(\mathrm{P}_{0}\right)$, a renda familiar advinda do trabalho per capita (W), as transferências governamentais médias de renda per capita (T) e o índice de concentração de renda de Gini (G). Portanto, este artigo decompõe a elasticidade-renda da pobreza em duas partes,

4 A equação (4) também pode ser expressa como: $\min \frac{1}{n} \sum_{i=1}^{n} \rho(\theta)\left(y-x_{i} \beta\right)$ em que $\rho$ é uma função check definida por: $\rho(\theta)\left\{\begin{array}{cc}\theta z, & z \geq 0 \\ (\theta-1) z, & z<0\end{array}\right.$, cujas soluções $k$-dimensionais foram definidas por Koenker e Basset (1978) como quantis de regressão, denotados por $\beta(\theta)$.

OLIVEIRA, C. A.; LIMA, F. S. Impacto do crescimento, da concentração da renda... 
uma parte considera os rendimentos obtidos do trabalho e a outra parte considera os rendimentos obtidos através de transferências governamentais de renda. O objetivo é avaliar separadamente os impactos do crescimento econômico e de políticas de transferência de renda na pobreza.

Os dados utilizados no artigo são fornecidos pelo Instituto Brasileiro de Geografia e Estatístico (IBGE) através dos censos demográficos de 1991 e 2000. Estes foram atualizados monetariamente para valores em reais de 2000. A linha de pobreza estabelecida é de pessoas com renda per capita inferior a $\mathrm{R} \$ 75,50$. Todas as variáveis acima foram obtidas considerando a compatibilização dos dados municipais feita pelo Atlas do Desenvolvimento Humano publicado pelo Programa das Nações Unidas para o Desenvolvimento (PNUD), construídos a partir dos dados dos censos demográficos de 1991 e 2000 realizados pelo Instituto Brasileiro de Geografia e Estatístico (IBGE).

No presente artigo, o primeiro passo foi a busca pela melhor forma funcional para a estimação das elasticidades. Este procedimento é sempre um desafio para o pesquisador devido à grande possibilidade de se cometer algum viés na especificação do modelo. Entretanto, os tradicionais critérios de informação de Akaike (AIC) e Bayesiano (BIC) e a verossimilhança (LL) dos modelos podem ser utilizados sempre que os modelos forem aninhados. É claro que sempre será desejável que o modelo escolhido possua algum fundamento teórico que justifique a escolha.

Tabela 1 - Avaliação da Especificação

\begin{tabular}{c|c|c|c}
\hline \multicolumn{5}{c}{ Variável dependente: $\mathbf{P}_{\mathbf{0}}$ em 1991 } \\
\hline & Modelo 1 & Modelo 2 & Modelo 3 \\
\hline $\mathrm{R}^{2}$ ajustado & 0.8885 & 0.8938 & 0.8938 \\
\hline Teste F & 4843.04 & 4771.71 & 4097.62 \\
\hline LL & 11351.94 & 11487.95 & 11489.39 \\
\hline AIC & -22687.87 & -22953.91 & -22952.77 \\
\hline BIC & -22634.96 & -22881.16 & -22866.80 \\
\hline \multicolumn{5}{|c|}{ Variável dependente: $\mathbf{P}_{\mathbf{0}}$ em 2000 } \\
\hline R ajustado & Modelo 1 & Modelo 2 & Modelo 3 \\
\hline Teste F & 0.8154 & 0.8188 & 0.8200 \\
\hline LL & 3186.13 & 2490.98 & 2191.81 \\
\hline AIC & 9493.01 & 9545.37 & 9564.29 \\
\hline BIC & -18970.03 & -19068.73 & -19102.58 \\
\hline
\end{tabular}

Fonte: Elaborada pelos autores. 
Para estimar as elasticidades renda e concentração da pobreza dos municípios do Brasil, foram testados basicamente três grupos de modelos. O primeiro grupo incluiu somente as variáveis independentes em nível, o segundo grupo acrescentou as variáveis na forma quadrática e o terceiro grupo acrescentou os termos cruzados. Em cada grupo foram testadas formas que excluíam as variáveis que não apresentavam significância estatística. O critério básico de escolha foi a forma funcional com a maior verossimilhança, menores critérios de informação e coeficientes significativos.

A Tabela 1 mostra os resultados obtidos através da estimação de um modelo de MQO com estimadores de White para variâncias e covariâncias e com dummies para as grandes regiões. O modelo com o melhor desempenho obtido segundo os critérios de informação foi um modelo do terceiro grupo, no entanto, este modelo apresenta alguns coeficientes não significativos, devido a problemas de multicolinearidade. Assim, seguindo os critérios estabelecidos, o modelo com o melhor desempenho obtido foi por um modelo completo do segundo grupo para a linha de pobreza estudada. Isto significa dizer que os modelos a serem estimados incluem as variáveis independentes tanto em nível quanto em suas formas quadráticas, ou seja, estima-se o seguinte modelo econométrico:

$$
P_{0 i}=\beta_{0}+\beta_{1} W_{i}+\beta_{2} G_{i}+\beta_{3} T_{i}+\beta_{4}\left(W_{i}\right)^{2}+\beta_{5}\left(G_{i}\right)^{2}+\beta_{6}\left(T_{i}\right)^{2}+\varepsilon_{i}
$$

Onde todos os modelos estimados utilizaram os dados em logaritmos. Um modelo que apresenta termos quadráticos apresenta algumas vantagens. Em primeiro lugar, permite testar a existência de não linearidades no modelo e, em segundo lugar, ajuda a capturar uma heterogeneidade existente, pois permite estimar as elasticidades para cada município em cada ponto no tempo. Utilizando este modelo, as elasticidades da renda e da concentração de renda para cada município $i$ no tempo $t=1991,2000$ serão, respectivamente:

$$
\begin{aligned}
& \varepsilon_{\mathrm{Wi}}=\beta_{1}+2 \beta_{4} \log W_{i} \\
& \varepsilon_{G i}=\beta_{2}+2 \beta_{5} \log G_{i} \\
& \varepsilon_{T i}=\beta_{3}+2 \beta_{6} \log T_{i}
\end{aligned}
$$

\section{Resultados}

Nesta seção são apresentados e analisados os principais resultados obtidos nas regressões quantílicas e os cálculos das elasticidades. Na Tabela 2 são apresentados os resultados obtidos na estimação nos quantis da equação (5) para os anos de 1991 e 2000 para os 5507 municípios brasileiros. Além da especificação dada por (5), os modelos estimados 
incluem dummies para as grandes regiões com o objetivo de captar a heterogeneidade não observada. Os erros padrões são obtidos via bootstrap com 20 replicações da distribuição original.

Os sinais observados são os esperados e os modelos mostram um bom ajustamento. ${ }^{5}$ No entanto, cabe observar que as regressões quantílicas apresentam alguns coeficientes não significativos nos extremos de suas distribuições condicionais. É possível observar que a concentração de renda contribui para aumentar o número de famílias com renda abaixo da linha de pobreza em todos os quantis nos dois períodos estudados e que a contribuição da renda em suas duas formas é a priori ambígua e seu sinal dependerá do nível de renda do município.

Tabela 2 - Resultados das Regressões

\begin{tabular}{|c|c|c|c|c|c|c|c|c|}
\hline \multicolumn{9}{|c|}{1991} \\
\hline Quantil & W & G & $\mathrm{T}$ & $W^{2}$ & $G^{2}$ & $\mathrm{~T}^{2}$ & Constante & Pseudo $\mathrm{R}^{2}$ \\
\hline 10 & $\begin{array}{l}-0.0840 \\
(0.0424)\end{array}$ & $\begin{array}{l}0.9413 \\
(0.1686)\end{array}$ & $\begin{array}{l}-0.0592 \\
(0.0104)\end{array}$ & $\begin{array}{l}-0.0750 \\
(0.0114)\end{array}$ & $\begin{array}{l}0.5656 \\
(0.2899)\end{array}$ & $\begin{array}{l}0.0213 \\
(0.0064)\end{array}$ & $\begin{array}{l}2.3517 \\
(0.0527)\end{array}$ & 0.7220 \\
\hline 25 & $\begin{array}{l}-0.2605 \\
(0.0216)\end{array}$ & $\begin{array}{l}1.1144 \\
(0.1387)\end{array}$ & $\begin{array}{l}-0.0652 \\
(0.0055)\end{array}$ & $\begin{array}{l}-0.0246 \\
(0.0050)\end{array}$ & $\begin{array}{l}0.8871 \\
(0.2354)\end{array}$ & $\begin{array}{l}0.0213 \\
(0.0033)\end{array}$ & $\begin{array}{l}2.5420 \\
(0.0325)\end{array}$ & 0.7405 \\
\hline 50 & $\begin{array}{l}-0.4458 \\
(0.0195)\end{array}$ & $\begin{array}{l}1.2638 \\
(0.0961)\end{array}$ & $\begin{array}{l}-0.0759 \\
(0.0074)\end{array}$ & $\begin{array}{l}0.0294 \\
(0.0049)\end{array}$ & $\begin{array}{l}1.1422 \\
(0.1677)\end{array}$ & $\begin{array}{l}0.0253 \\
(0.0042)\end{array}$ & $\begin{array}{l}2.7371 \\
(0.0235)\end{array}$ & 0.7431 \\
\hline 75 & $\begin{array}{l}-0.5905 \\
(0.0298)\end{array}$ & $\begin{array}{l}1.2918 \\
(0.1038)\end{array}$ & $\begin{array}{l}-0.0720 \\
(0.0116)\end{array}$ & $\begin{array}{l}0.0758 \\
(0.0085)\end{array}$ & $\begin{array}{l}1.2103 \\
(0.1704)\end{array}$ & $\begin{array}{l}0.0204 \\
(0.0069)\end{array}$ & $\begin{array}{l}2.8594 \\
(0.0228)\end{array}$ & 0.7126 \\
\hline 90 & $\begin{array}{l}-0.7242 \\
(0.0357)\end{array}$ & $\begin{array}{l}1.2780 \\
(0.0879)\end{array}$ & $\begin{array}{l}-0.0778 \\
(0.0230)\end{array}$ & $\begin{array}{l}0.1213 \\
(0.0095)\end{array}$ & $\begin{array}{l}1.1984 \\
(0.1495)\end{array}$ & $\begin{array}{l}0.0197 \\
(0.0103)\end{array}$ & $\begin{array}{l}2.9642 \\
(0.0350)\end{array}$ & 0.6658 \\
\hline \multicolumn{9}{|c|}{2000} \\
\hline Quantil & W & G & $\mathrm{T}$ & $\mathrm{W}^{2}$ & $\mathrm{G}^{2}$ & $\mathrm{~T}^{2}$ & Constante & Pseudo $R^{2}$ \\
\hline 10 & $\begin{array}{l}0.2069 \\
(0.0214)\end{array}$ & $\begin{array}{l}0.1721^{*} \\
(0.2563)\end{array}$ & $\begin{array}{l}-0.0776 \\
(0.0291)\end{array}$ & $\begin{array}{l}-0.1276 \\
(0.0064)\end{array}$ & $\begin{array}{l}-1.0795 \\
(0.5415)\end{array}$ & $\begin{array}{l}0.0178^{*} \\
(0.0130)\end{array}$ & $\begin{array}{l}1.9114 \\
(0.0449)\end{array}$ & 0.6275 \\
\hline 25 & $\begin{array}{l}0.0963 \\
(0.0207)\end{array}$ & $\begin{array}{l}0.1058^{*} \\
(0.1109)\end{array}$ & $\begin{array}{l}-0.1278 \\
(0.0197)\end{array}$ & $\begin{array}{l}-0.0898 \\
(0.0065)\end{array}$ & $\begin{array}{l}-1.1601 \\
(0.2356)\end{array}$ & $\begin{array}{l}0.0359 \\
(0.0080)\end{array}$ & $\begin{array}{l}2.0227 \\
(0.0242)\end{array}$ & 0.6456 \\
\hline 50 & $\begin{array}{l}-0.0582 \\
(0.0359)\end{array}$ & $\begin{array}{l}0.2445 \\
(0.1574)\end{array}$ & $\begin{array}{l}-0.1522 \\
(0.0281)\end{array}$ & $\begin{array}{l}-0.0404 \\
(0.0103)\end{array}$ & $\begin{array}{l}-0.8251 \\
(0.3282)\end{array}$ & $\begin{array}{l}0.0424 \\
(0.0116)\end{array}$ & $\begin{array}{l}2.1869 \\
(0.0249)\end{array}$ & 0.6515 \\
\hline 75 & $\begin{array}{l}-0.2099 \\
(0.0239)\end{array}$ & $\begin{array}{l}0.5149 \\
(0.1523)\end{array}$ & $\begin{array}{l}-0.2171 \\
(0.0515)\end{array}$ & $\begin{array}{l}0.0078^{*} \\
(0.0070)\end{array}$ & $\begin{array}{c}-0.1729^{*} \\
(0.3044)\end{array}$ & $\begin{array}{l}0.0647 \\
(0.0209)\end{array}$ & $\begin{array}{l}2.3872 \\
(0.0343)\end{array}$ & 0.6156 \\
\hline 90 & $\begin{array}{l}-0.3700 \\
(0.0395)\end{array}$ & $\begin{array}{l}0.8816 \\
(0.2904)\end{array}$ & $\begin{array}{l}-0.2360 \\
(0.0546)\end{array}$ & $\begin{array}{c}0.0602 \\
(0117)\end{array}$ & $\begin{array}{l}0.6481 * \\
(0.5866)\end{array}$ & $\begin{array}{l}0.0655 \\
(0.0219)\end{array}$ & $\begin{array}{l}2.5800 \\
(0.0485)\end{array}$ & 0.5662 \\
\hline
\end{tabular}

Notas: Erro padrão entre parênteses. * não significativo a 5\%.

Fonte: Elaborada pelos autores.

$5 \quad$ Muito embora este pseudo $\mathrm{R}^{2}$ deva ser interpretado com cautela, pois é somente uma aproximação de um ajustamento, este difere do tradicional obtido em MQO e é obtido ao subtrair a razão da mínima soma dos desvios ponderados e a soma bruta dos desvios ponderados de um. 
Interpretações adicionais são dificultadas pela não linearidade do modelo estimado. Entretanto, uma combinação dos valores dos coeficientes estimados e os valores das rendas advindas do trabalho e de transferências e concentração de renda, permite os cálculos das elasticidades renda e concentração de renda da pobreza em acordo com equação 6. As Figuras 1, 2, 3, 4, 5 e 6 apresentam as elasticidades estimadas para o Brasil em cada quantil e em cada período com um intervalo de confiança de $95 \%$. São apresentados ainda os valores estimados por MQO e seus respectivos intervalos de confiança de mesmo tamanho.

Os resultados obtidos mostram que há uma heterogeneidade nas elasticidades que um modelo de MQO não consegue captar. Em alguns casos, a elasticidade estimada nos quantis está fora do intervalo de confiança do estimador de MQO, o que é um indício de viés nestes estimadores. Há, inclusive, inversão de sinais, pois as elasticidades-renda (de transferências) apresentam sinal positivo quando estimados por MQO, mas apresentam sinal negativo quando estimados por regressões quantílicas. Este viés poderia levar a concluir que transferências de renda são ineficazes na redução da pobreza, o que nem sempre é verdade, pois apesar do intervalo de confiança permitir valores tanto positivos quanto negativos, o seu impacto em toda a distribuição condicional é negativo.

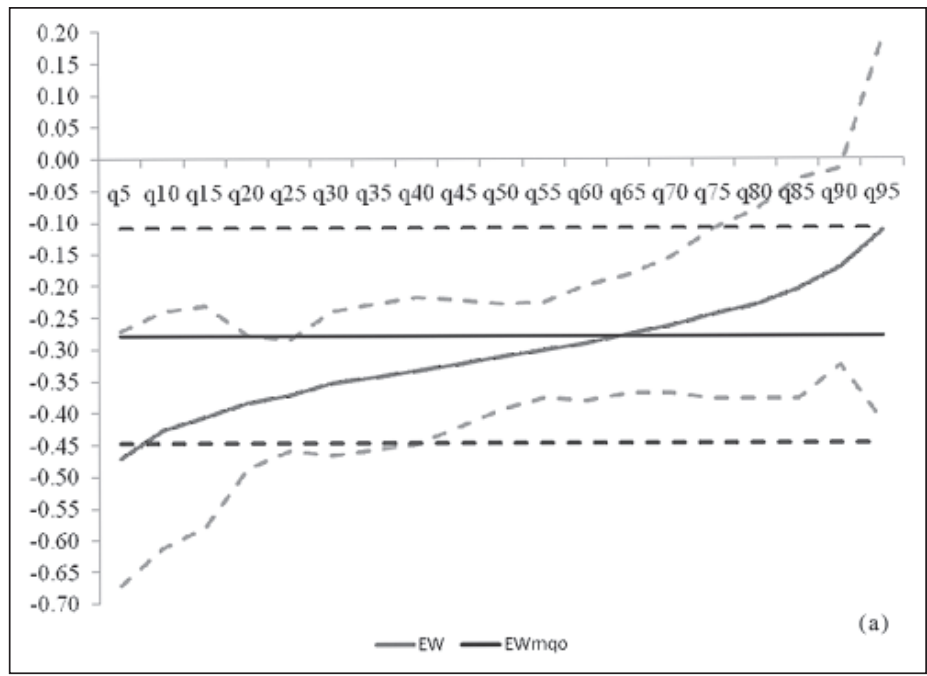

Figura 1 - Elasticidades Estimadas para o Brasil por Quintil ( $1^{\text {a }}$ de 6$)$ Nota: Elasticidade-renda (do trabalho) em 1991. Fonte: Elaborada pelos autores. 


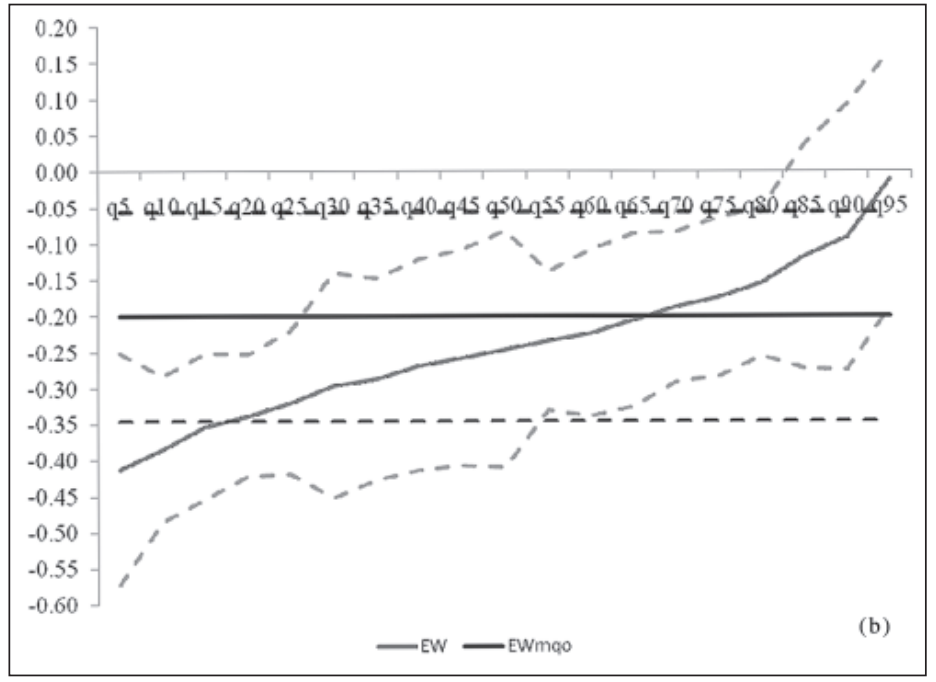

Figura 2 - Elasticidades Estimadas para o Brasil por Quintil (2 $2^{\mathrm{a}}$ de 6) Nota: Elasticidade-renda (do trabalho) em 2000.

Fonte: Elaborada pelos autores.

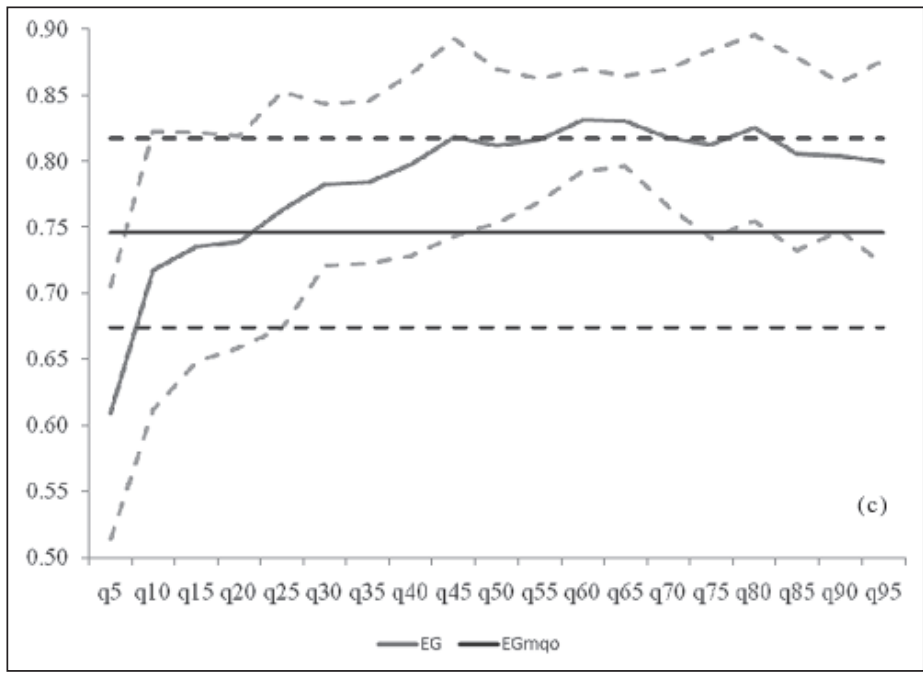

Figura 3 - Elasticidades Estimadas para o Brasil por Quintil ( $3^{\mathrm{a}}$ de 6$)$ Nota: Elasticidade-concentração em 1991.

Fonte: Elaborada pelos autores. 


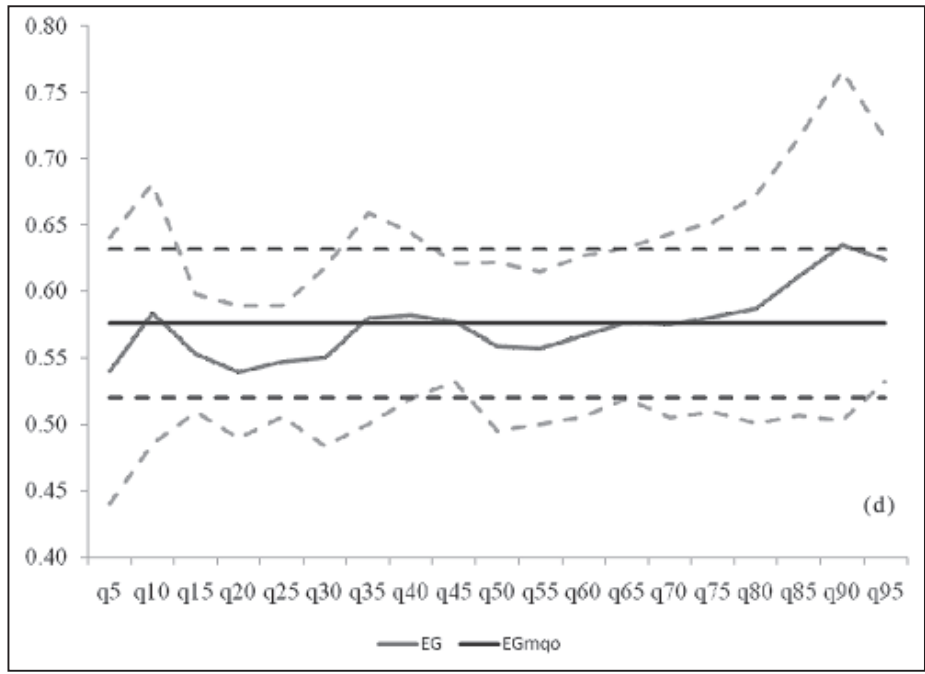

Figura 4 - Elasticidades Estimadas para o Brasil por Quintil ( $4^{\mathrm{a}}$ de 6)

Nota: Elasticidade-concentração em 2000.

Fonte: Elaborada pelos autores.

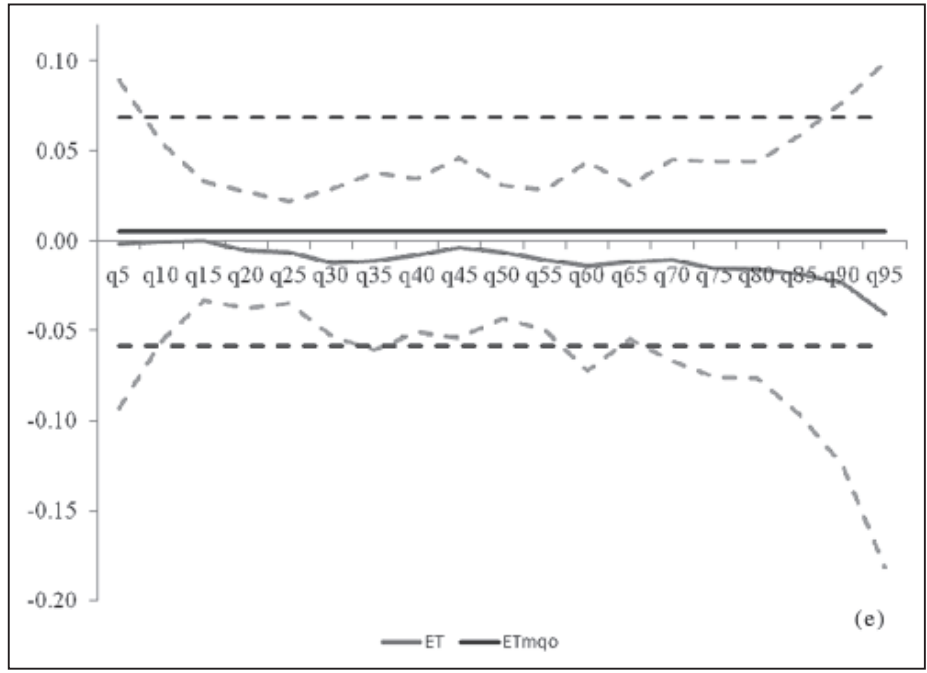

Figura 5 - Elasticidades Estimadas para o Brasil por Quintil (5 a de 6)

Nota: Elasticidade-renda (de transferências) em 1991.

Fonte: Elaborada pelos autores.

OLIVEIRA, C. A.; LIMA, F. S. Impacto do crescimento, da concentração da renda... 


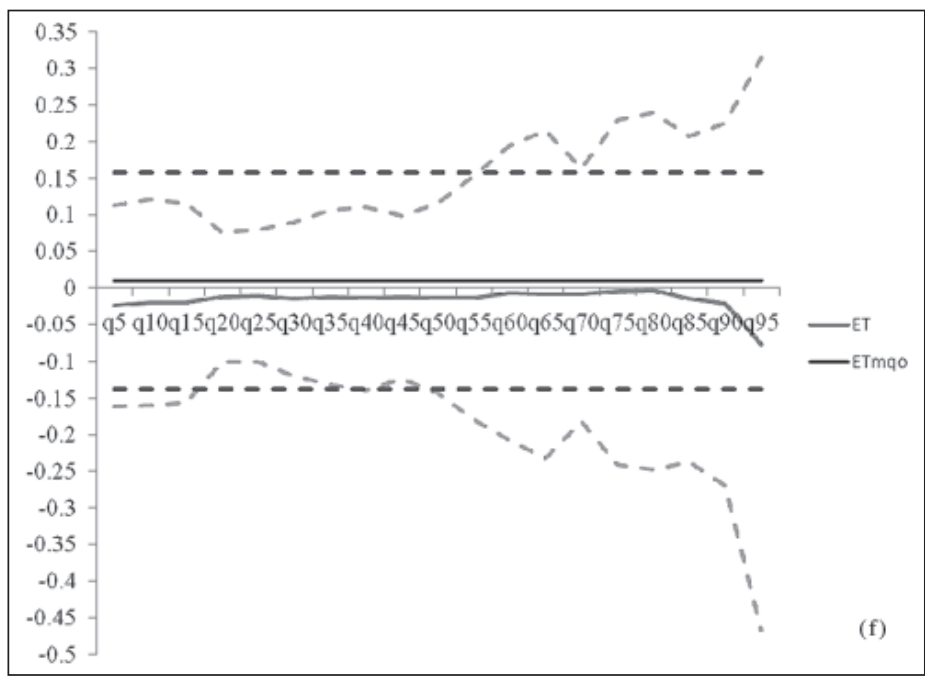

Figura 6 - Elasticidades Estimadas para o Brasil por Quintil ( $6^{\mathrm{a}}$ de 6$)$

Nota: Elasticidade-renda (de transferências) em 2000.

Fonte: Elaborada pelos autores.

Os estimadores nos quantis permitem confirmar a teoria de que as elasticidades diminuem com o aumento do nível de pobreza. Este resultado tem uma implicação de política importante, pois municípios com muita pobreza têm que crescer economicamente e diminuir a concentração de renda mais do que um município com pouca pobreza para poderem reduzir o seu número de pobres. As Figuras permitem ainda observar a forma como as elasticidades mudam ao longo da distribuição condicional e no tempo. As elasticidades-renda (do trabalho) e da concentração de renda crescem na medida em que o município possui menos pobres. No entanto, há uma estabilidade da elasticidade concentração na segunda metade da distribuição condicional em 1991 e alguma oscilação em torno da elasticidade estimada por MQO na primeira metade da distribuição condicional de 2000. As elasticidades renda (de transferências) não apresentam uma estabilidade nos dois períodos estudados. Apesar de apresentarem uma pequena variação, mostram uma tendência decrescente com o quantil em 1991 e um formato de "U" invertido em 2000. Isto significa que o impacto das transferências de renda foi maior em municípios com mais pobres, o que do ponto de vista de política pública é algo desejável.

As Figuras mostram que as elasticidades-renda (do trabalho) diminuíram entre os anos de 1991 e 2000 em todos os quantis. Em termos relativos, a elasticidade-renda de um município situado no décimo quantil aumentou quando comparado à elasticidade dos municípios que se encontram no 
nonagésimo quantil. Isto significa que o crescimento econômico tornou-se menos eficiente em reduzir a pobreza, principalmente nos municípios em que a incidência de pobreza é mais acentuada. O que pode significar que no caso brasileiro o crescimento econômico seja pouco eficiente na redução da pobreza, dada a forma como este acréscimo na renda é distribuído, não permitindo a inclusão dos mais pobres. Isto fica mais evidente em municípios com muitos pobres. Em 2000, por exemplo, um aumento em 9\% da renda média de um município situado no nonagésimo quantil reduz a pobreza em aproximadamente apenas 1\%. Estas elasticidades calculadas estão bem abaixo das calculadas em outros trabalhos, tais como Marinho e Soares (2003) e Hoffman (2005), que não consideram a heterogeneidade existente entre os municípios.

A elasticidade concentração de renda da pobreza apresenta valores mais semelhantes a outros trabalhos do que a elasticidade-renda. Os resultados mostram que esta elasticidade reduziu em todos os quantis do ano de 1991 para 2000. O que implica que esta se tornou menos eficiente na redução da pobreza. Provavelmente isto ocorreu devido a uma redução da mesma em todos os quantis da distribuição. Entretanto, estas elasticidades permanecem altas e reafirmam a necessidade de políticas que objetivem a redução da desigualdade. A elasticidade concentração da renda é sempre superior à elasticidade da renda em todos os quantis nos dois períodos estimados, o que afirma o seu relevante papel na redução da pobreza.

A redução da desigualdade mostra-se um caminho interessante para a redução da pobreza, uma vez que um aumento de 1\% na desigualdade de um município situado no décimo quantil implica na necessidade de um aumento da renda média em 1,68\% e 1,51\% para que não aumentasse a pobreza em 1991 e 2000, respectivamente. Já no nonagésimo quantil, a renda teria que aumentar 4,71\% e 6,98\% em 1991 e 2000, respectivamente. Este raciocínio vai de encontro ao que propõe Kakwani (1993, p. 133):

[...] a greater emphasis should be placed on the growth oriented policies which at least maintain the income share of the poor... if the income inequality deteriorates during the course of a country's economic growth, the poverty may even increase because the poverty measures are considerably more elastic for changes in inequality [...]

As altas elasticidades calculadas neste artigo para a elasticidade-concentração e as baixas para elasticidade-renda corroboram com a adequação das ideias do autor também no caso brasileiro. O crescimento econômico pode ser insuficiente para a redução da pobreza em vários municípios devido à forma como a mesma é distribuída neste município. 
As elasticidades-renda (de transferências governamentais) com relação à pobreza apresentam um efeito ambíguo na pobreza. As elasticidades estimadas nos quantis para o Brasil são sempre negativas, no entanto, o seu intervalo de confiança permite valores positivos em todos os quantis nos dois períodos estudados. A evolução desta elasticidade nos dois períodos difere de acordo com a posição na distribuição condicional. Houve um aumento da elasticidade na primeira metade da distribuição condicional e uma redução na segunda metade até o nonagésimo quantil e um aumento no nonagésimo quinto quantil. Isto significa que as transferências de renda tornaram-se mais eficientes na redução da pobreza nos municípios com poucos pobres, mas houve um acréscimo bastante significativo no último decil da distribuição condicional, pois a elasticidade cresceu de 4,09 para 7,6 . Este resultado demonstra que este tipo de política pública pode ser eficiente na redução da pobreza quando atinge os municípios que realmente possuem a maior parte de sua população abaixo da linha de pobreza proposta. No entanto, fica claro que seu impacto deve ser avaliado caso a caso, pois as baixas elasticidades encontradas e o impacto ambíguo podem não recomendar o seu uso.

Estas considerações a respeito das elasticidades, sobre os três tipos de políticas para a redução da pobreza, a saber, crescimento econômico, redução da desigualdade de renda e aumento das transferências governamentais e as suas eficiências na redução da pobreza desconsideram os seus custos de execução e eventuais distorções causadas por interferência governamental. Cabe então, ao gestor de políticas públicas, avaliar quais são as alternativas mais eficazes a um menor custo. Estas políticas podem inclusive ser diferenciadas por municípios, já que estes apresentam situações (elasticidades) que podem ser muito distintas. Esta ideia pode ser ilustrada pela Tabela 3.

Tabela 3 - Elasticidades Estimadas para os 10 Maiores Municípios Brasileiros, 1991 e 2000

\begin{tabular}{l|c|c|c|c|c|c|c|c|c}
\hline \multicolumn{1}{c}{ Município } & $\varepsilon_{\mathrm{W}}$ & $\mathrm{li}$ & $\mathrm{ls}$ & $\varepsilon_{\mathrm{G}}$ & li & ls & $\varepsilon_{\mathrm{T}}$ & \multicolumn{1}{c}{ li } & ls \\
\hline São Paulo (SP) & -0.2901 & -0.4816 & -0.0810 & 0.6885 & 0.6565 & 0.6744 & 0.0074 & -0.0129 & 0.0111 \\
\hline Rio de Janeiro (RJ) & -0.2967 & -0.4647 & -0.1083 & 0.7734 & 0.6985 & 0.7634 & 0.0189 & -0.0040 & 0.0207 \\
\hline Salvador (BA) & -0.3068 & -0.4389 & -0.1501 & 0.8364 & 0.7297 & 0.8295 & 0.0013 & -0.0177 & 0.0058 \\
\hline Belo Horizonte (MG) & -0.2971 & -0.4636 & -0.1101 & 0.7734 & 0.6985 & 0.7634 & 0.0089 & -0.0118 & 0.0123 \\
\hline Fortaleza (CE) & -0.3114 & -0.4272 & -0.1690 & 0.8364 & 0.7297 & 0.8295 & -0.0022 & -0.0204 & 0.0029 \\
\hline Brasília (DF) & -0.2920 & -0.4768 & -0.0888 & 0.7734 & 0.6985 & 0.7634 & 0.0005 & -0.0183 & 0.0052 \\
\hline Curitiba (PR) & -0.2944 & -0.4704 & -0.0990 & 0.6707 & 0.6476 & 0.6556 & 0.0073 & -0.0130 & 0.0110 \\
\hline Recife (PE) & -0.3062 & -0.4403 & -0.1477 & 0.8665 & 0.7446 & 0.8611 & 0.0079 & -0.0125 & 0.0115 \\
\hline
\end{tabular}


(continuação)

\begin{tabular}{|c|c|c|c|c|c|c|c|c|c|}
\hline Manaus (AM) & -0.3061 & -0.4406 & -0.1473 & 0.7061 & 0.6652 & 0.6928 & -0.0127 & -0.0286 & -0.0060 \\
\hline Porto Alegre (RS) & -0.292 & -0.4765 & -0.0891 & 0.7061 & 0.6652 & 0.6928 & 0.0201 & -0.0031 & 0.0217 \\
\hline \multicolumn{10}{|c|}{2000} \\
\hline Município & $\varepsilon_{\mathrm{W}}$ & li & ls & $\varepsilon_{\mathrm{G}}$ & li & ls & $\varepsilon_{\mathrm{T}}$ & li & ls \\
\hline São Paulo (SP) & -0.2718 & -0.4668 & -0.052 & 0.5871 & 0.6203 & 0.6125 & 0.0040 & -0.0120 & 0.0057 \\
\hline Rio de Janeiro (RJ) & -0.2669 & -0.4515 & -0.0592 & 0.5871 & 0.6203 & 0.6125 & 0.0208 & -0.0050 & 0.0316 \\
\hline Salvador (BA) & -0.2489 & -0.3946 & -0.0861 & 0.5423 & 0.5617 & 0.6477 & -0.0085 & -0.0172 & 0.0136 \\
\hline Belo Horizonte (MG) & -0.2675 & -0.4535 & -0.0583 & 0.5871 & 0.6203 & 0.6125 & 0.0138 & -0.0079 & 0.0208 \\
\hline Fortaleza (CE) & -0.2472 & -0.3892 & -0.0886 & 0.5423 & 0.5617 & 0.6477 & -0.0126 & -0.0190 & 0.0199 \\
\hline Brasília (DF) & -0.2727 & -0.4697 & -0.0506 & 0.5643 & 0.5906 & 0.6304 & 0.0068 & -0.0109 & 0.0099 \\
\hline Curitiba (PR) & -0.2731 & -0.4711 & -0.0499 & 0.6226 & 0.6668 & 0.5846 & 0.0083 & -0.0102 & 0.0123 \\
\hline Recife (PE) & -0.2530 & -0.4075 & -0.0800 & 0.5209 & 0.5337 & 0.6645 & 0.0068 & -0.0108 & 0.0101 \\
\hline Manaus (AM) & -0.2415 & -0.3713 & -0.0971 & 0.5643 & 0.5906 & 0.6304 & -0.0333 & -0.0377 & 0.0520 \\
\hline Porto Alegre (RS) & -0.2758 & -0.4794 & -0.046 & 0.5987 & 0.6356 & 0.6033 & 0.0266 & -0.0025 & 0.0406 \\
\hline
\end{tabular}

Fonte: Elaborada pelos autores.

A Tabela 3 mostra as elasticidades calculadas para os maiores municípios brasileiros em 1991 e 2000. Estas foram calculadas de acordo com os parâmetros estimados em cada quantil em cada ano. Como não há um parâmetro estimado único, a Tabela 3 apresenta o valor calculado a partir da mediana e constrói um intervalo de confiança semiparamétrico baseado nos valores estimados nos decis inferiores e superiores da distribuição condicional.

Como pode ser visto na Tabela 3, todas as elasticidades-renda (do trabalho) e da concentração da renda diminuíram em relação a 1991. Esta redução foi maior nas capitais nordestinas, o que implica na redução da eficiência destas políticas na redução da pobreza justamente nas regiões mais pobres do País, entretanto, as elasticidades-concentração ainda permaneceram bastante altas.

O intervalo de confiança para a elasticidade-renda (do trabalho) mostra a dificuldade em avaliar os impactos do crescimento econômico na redução da pobreza nos maiores municípios do Brasil. Em Porto Alegre, em 2000, a maior elasticidade do período, um aumento da renda do trabalho em $10 \%$ poderia reduzir a pobreza em um intervalo entre 4,6\% e 47,94\%. Há claramente pouca eficiência nas estimativas. Já as estimações para a elasticidade-renda (de transferências governamentais) são mais precisas com relação ao intervalo, mas são ambíguas com respeito ao seu sinal. Somente Manaus em 1991 e 2000 e Salvador e Fortaleza em 2000 possuem um intervalo que inclui somente valores de mesmo sinal (negativo). $\mathrm{Na}$ maior parte dos municípios, os valores são próximos a zero e positivos. $\mathrm{O}$ que implica um aumento da pobreza com o incremento das transferências governamentais nestes municípios. Como foi visto anteriormente, 
a maior eficiência deste tipo de política encontra-se em municípios com muitos pobres (a partir do nono decil), o que não é o caso de nenhum destes municípios estudados.

Por sua vez, as elasticidades-concentração são bastante altas e apresentam menor variabilidade. Estas são maiores nos municípios nordestinos em 1991, porém são mais semelhantes em todos os municípios em 2000. Os resultados mostram que esta mantém aproximadamente uma relação de 2 para 1 com a elasticidade renda, sendo que esta relação foi reduzida em praticamente todos os municípios estudados com a exceção de Manaus e Curitiba, que aumentaram esta relação. Isto significa dizer que um aumento de $1 \%$ na desigualdade de renda destes municípios deveria ser compensado por um aumento de aproximadamente $2 \%$ na renda para que não houvesse aumento da pobreza no município. O que reafirma a necessidade de políticas nestes municípios que reduzam a desigualdade como forma de combate à pobreza.

Por fim, como estas elasticidades estimadas possuem sinais contrários, na verdade estas atuam como forças contrárias que irão determinar o impacto resultante na pobreza de mudanças na renda, na desigualdade de renda e nas transferências governamentais. Desta forma, as elasticidades apresentadas na Tabela 3 permitem estimar a contribuição da renda, da concentração da renda e das transferências governamentais na variação da pobreza $\left(\mathrm{P}_{0}\right)$ observada no período. Os resultados são apresentados na Tabela 4.

Tabela 4 - Contribuição do Crescimento Econômico, da Desigualdade de Renda e das Transferências Governamentais na Redução da Pobreza dos 10 Maiores Municípios Brasileiros no Período 1991-2000.

\begin{tabular}{l|c|c|c}
\hline \multicolumn{1}{c|}{ Município } & $\begin{array}{c}\text { Contribuição do } \\
\text { Crescimento } \\
\text { Econômico }\end{array}$ & $\begin{array}{c}\text { Contribuição da } \\
\text { Desigualdade da } \\
\text { Renda }\end{array}$ & $\begin{array}{c}\text { Contribuição das } \\
\text { Transferências } \\
\text { Governamentais }\end{array}$ \\
\hline São Paulo (SP) & 0.0079 & 0.0683 & 0.0033 \\
\hline Rio de Janeiro (RJ) & -0.0277 & 0.0112 & 0.0095 \\
\hline Salvador (BA) & 0.0065 & 0.0106 & -0.0018 \\
\hline Belo Horizonte (MG) & -0.0385 & 0.0112 & 0.0105 \\
\hline Fortaleza (CE) & -0.0316 & 0.0106 & -0.0041 \\
\hline Brasília (DF) & -0.0211 & 0.0329 & 0.0048 \\
\hline Curitiba (PR) & -0.0568 & 0.0470 & 0.0061 \\
\hline Recife (PE) & -0.0204 & 0.0104 & 0.0049 \\
\hline Manaus (AM) & 0.0627 & 0.0780 & -0.0100 \\
\hline Porto Alegre (RS) & -0.0503 & 0.0458 & 0.0149 \\
\hline
\end{tabular}

Fonte: Elaborada pelos autores a partir das médias das elasticidades estimadas. 
A Tabela 4 mostra que o aumento na desigualdade de renda contribuiu para o aumento da pobreza em todas as grandes cidades brasileiras na década de noventa. Entretanto, cabe ressaltar que o aumento da renda (crescimento econômico) pode atuar em sentido contrário e reduzir a pobreza. Infelizmente, estes não foram os casos das cidades de São Paulo, Salvador, Manaus. Este último aumentou a sua pobreza em mais de 6\% devido à redução da renda e aproximadamente $8 \%$ devido ao aumento da desigualdade. Neste caso, as transferências governamentais de renda foram insuficientes para neutralizar estes incrementos, pois contribuíram para a redução de apenas $1 \%$ na pobreza do município.

Os municípios de Curitiba e Porto Alegre apresentaram o maior impacto positivo do crescimento econômico na redução da pobreza. No entanto, estas também tiveram entre os maiores impactos negativos do aumento da desigualdade da renda, o que reafirma a ideia de que o crescimento econômico pode ser insuficiente para a redução da pobreza se não houver uma mudança na forma como a renda é distribuída no município. No caso destes municípios, as transferências de renda ainda contribuíram para o aumento da pobreza gerando um efeito líquido praticamente nulo na redução da pobreza.

As transferências governamentais de renda mostraram pouca contribuição para a redução da pobreza nos municípios estudados. Somente em Salvador (0,18\%), Fortaleza $(0,41 \%)$ e Manaus $(0,1 \%)$ este tipo de política teve o efeito desejado, mesmo que estas transferências tenham sido aumentadas nestes municípios em 48,8\%, 55,86\% e 43,48\%, respectivamente. Cabe ressaltar que esta análise não considera a distância que os pobres destes municípios estavam no período inicial. De qualquer forma, este fato associado aos impactos positivos (de aumento da pobreza) nos demais municípios estudados talvez seja um indício de desvio do foco dos programas de combate à pobreza que utilizam a transferência simples de renda como instrumento.

\section{Considerações Finais}

Este artigo utiliza regressão quantílica para estimar as elasticidades da renda (do trabalho e de transferências governamentais) e a concentração da renda em relação à pobreza dos municípios brasileiros nos anos de 1991 e 2000.

Os principais resultados encontrados confirmam outras investigações anteriores que mostram que a distribuição da renda tem maior impacto sobre a pobreza do que o crescimento da renda média. Os resultados mostram também que as elasticidades renda e concentração da pobreza estão diminuindo em todos os grupos de municípios. Além 
disto, pode-se demonstrar que nos municípios com maior incidência de pobreza, esta elasticidade reduziu mais rápido do que nos municípios com menos pobres.

As políticas de distribuição de renda mostram-se mais eficazes na redução da pobreza. Pode-se notar ainda que os seus efeitos se tornaram menos acentuados com o tempo. O efeito da distribuição da renda sobre a pobreza é um fenômeno que precisa ser mais bem estudado para que permita a elaboração de políticas mais eficientes. A redução da desigualdade, assim como a redução da pobreza, é sempre um objetivo desejável, ainda mais no Brasil, que apresenta uma das maiores desigualdades de renda do mundo. No entanto, atingir este objetivo pode servir de justificativa para políticas intervencionistas cuja eficiência é discutível e cujos efeitos colaterais não são medidos quando da sua implementação.

O efeito das transferências, apesar de ser negativo (reduz pobreza) na maioria dos casos, é relativamente pequeno, mostrando uma elevação da sua eficácia no período estudado principalmente nos municípios com muitos pobres. Porém, sempre apresentaram um impacto menor na pobreza do que o crescimento econômico. Sempre vale lembrar que os três tipos de políticas para a redução da pobreza, a saber, crescimento econômico, redução da desigualdade de renda e aumento das transferências governamentais, possuem custos de execução e impactos diferentes, cabendo então, ao gestor de políticas públicas avaliar quais são as alternativas mais eficazes a um menor custo. Estas políticas podem ser diferenciadas por municípios e esta talvez seja a maior contribuição deste artigo. Este contribui, pois, com o debate, mostrando que os efeitos das políticas de renda podem ser avaliados por município e que não é correto pensar em uma política de combate à pobreza única para o País ou mesmo para um Estado. Para cada nível de renda e nível de concentração de renda deve-se ter políticas específicas para reduzir a pobreza mais eficientemente. Entretanto, é válido concluir afirmando que a pobreza é um problema complexo que implica, necessariamente, soluções complexas. Não existe uma causa única e nem uma solução única.

\section{Referências}

BARRETO, F. A. Crescimento econômico, pobreza e desigualdade de renda: o que sabemos sobre eles. Ensaios sobre pobreza. LEP/CAEN/UFC. Dez. 2005.

BARROS, R.P.; MENDONÇA, R. O impacto do crescimento econômico e de reduções no grau de desigualdade sobre a pobreza. Texto para Discussão: IPEA, Rio de Janeiro, n.528, 1997.

BUCHINSKY, M. Recent advances in quantile regression models: a practical guideline for empirical research. Journal of Human Resources, n.r33, p. 88-126, 1998. 
BUHAI, S. Quantile Regression: overview and selected applications. Mimeo: Tinbergen Institute, 2004.

CHEN, S.; WANG, Y. China's growth and poverty reduction: recent trends between 1990 and 1999. World Bank Working Paper, 2001.

FOSTER, J.; GREER, J.; THORBECKE, E. A class of decomposable poverty measures. Econometrica, n.52, p. 761-776, 1984.

HOFFMAN, R. Distribuição de renda: medidas de desigualdade e pobreza. São Paulo: Editora da USP, 1998.

. Elasticidade da pobreza em relação à renda média e a desigualdade no Brasil e nas Unidades da Federação. Revista Economia, v. 6, n.2, p. 255-289, 2005.

KAKWANI, N. Poverty and economic growth: with application to Côte d'Ivoire. Review of Income and Wealth, n.39, p. 121-139, 1993.

KAKWANI, N.; PERNIA, E. What is pro-poor Growth? Asian Development Review, v. 16, n.1, 1-22, 2000.

KOENKER, R.; BASSETT, G. Regression Quantiles. Econometrica, n. 46, p. 33-50, 1978.

KOENKER, R.; HALLOCK, K. Quantile Regression. Journal of Economic Perspectives, n.15, p.143-156, 2001.

LOPEZ, H.; SERVEN, L. The mechanics of growth-poverty-inequality relationship. Mimeo: The World Bank, 2004.

MARINHO, E.; SOARES, F. Impacto do crescimento econômico e da concentração de renda sobre a redução da pobreza nos estados brasileiros. In: ENCONTRO NACIONAL DE ECONOMIA, 31., 2003, Porto Seguro. Anais... Porto Seguro, 2003.

RAVALLION, M.; CHEN, S. Measuring pro-poor growth. Economic Letters, n.78, p. 93-99, 2003.

RAVALLION, M.; DATT, G. When is growth pro-poor? Evidence from the diverse experiences of India's states. Policy Research Working Paper: World Bank, Washington DC, n.2263, 1999.

SEN, A. The concept of development. In: HOLLIS, C.; SRINIVASAN, T. N. (Ed.). Handbook of Development Economics. Elsevier: North Holland, 1988. 


\section{Apêndice}

Tabela 5 - Estatística Descritiva das Variáveis Utilizadas

\begin{tabular}{c|c|c|c|c|c}
\hline Variável & Observações & Mínimo & Máximo & Média & $\begin{array}{c}\text { Desvio } \\
\text { Padrão }\end{array}$ \\
\hline Pobreza 1991 & 5507 & 11.78 & 87.29 & 48.60 & 9.97 \\
\hline Pobreza 2000 & 5507 & 15.76 & 83.03 & 47.09 & 10.73 \\
\hline $\begin{array}{c}\text { Renda per Capita } \\
1991\end{array}$ & 5507 & 17.66 & 501.54 & 103.87 & 63.85 \\
\hline $\begin{array}{c}\text { Renda per Capita } \\
\text { 2000 }\end{array}$ & 5507 & 6.79 & 659.38 & 117.16 & 76.54 \\
\hline $\begin{array}{c}\text { Transferências } \\
\text { Governamentais } \\
1991\end{array}$ & 5507 & 0.72 & 100.97 & 11.38 & 7.70 \\
\hline $\begin{array}{c}\text { Transferências } \\
\text { Governamentais } \\
\text { 2000 }\end{array}$ & 5507 & 2.21 & 161.32 & 26.95 & 14.40 \\
\hline $\begin{array}{c}\text { Índice } \\
\text { de Desigualdade } \\
\text { de Gini 1991 }\end{array}$ & 5507 & 0.35 & 0.79 & 0.53 & 0.06 \\
\hline $\begin{array}{c}\text { Índice } \\
\text { de Desigualdade } \\
\text { de Gini 2000 }\end{array}$ & 5507 & 0.36 & 0.82 & 0.56 & 0.06 \\
\hline
\end{tabular}

Fonte: Elaborada pelos autores.

Recebido em: 05/02/2009.

Aceito em: 22/09/2009. 